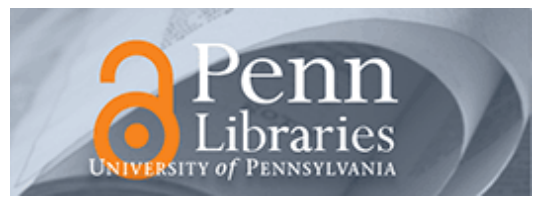

University of Pennsylvania

ScholarlyCommons

Operations, Information and Decisions Papers

Wharton Faculty Research

4-2011

\title{
The Value of Fast Fashion: Quick Response, Enhanced Design, and Strategic Consumer Behavior
}

Gerard. P. Cachon

University of Pennsylvania

Robert Swinney

Follow this and additional works at: https://repository.upenn.edu/oid_papers

Part of the Other Social and Behavioral Sciences Commons, and the Strategic Management Policy Commons

\section{Recommended Citation}

Cachon, G. P., \& Swinney, R. (2011). The Value of Fast Fashion: Quick Response, Enhanced Design, and Strategic Consumer Behavior. Management Sciecne, 57 (4), 778-795. http://dx.doi.org/10.1287/ mnsc. 1100.1303

This paper is posted at ScholarlyCommons. https://repository.upenn.edu/oid_papers/174

For more information, please contact repository@pobox.upenn.edu. 


\section{The Value of Fast Fashion: Quick Response, Enhanced Design, and Strategic Consumer Behavior}

\section{Keywords}

strategic consumer behavior, quick response, fast fashion, game theory

Disciplines

Other Social and Behavioral Sciences | Strategic Management Policy 


\title{
The Value of Fast Fashion: Quick Response, Enhanced Design, and Strategic Consumer Behavior
}

\author{
Gérard P. Cachon \\ The Wharton School, University of Pennsylvania, Philadelphia, PA 19104, cachon@wharton.upenn.edu \\ Robert Swinney \\ Graduate School of Business, Stanford University, Stanford, CA 94305, swinney@stanford.edu
}

September, 2010

\begin{abstract}
A fast fashion system combines quick response production capabilities with enhanced product design capabilities, to both design "hot" products that capture the latest consumer trends and exploit minimal production leadtimes to match supply with uncertain demand. We develop a model of such a system, and compare its performance to three alternative systems: quick response-only systems, enhanced design-only systems, and traditional systems (which lack both enhanced design and quick response capabilities). In particular, we focus on the impact of each of the four systems on "strategic" or forward-looking customer purchasing behavior, i.e., the intentional delay in purchasing an item at the full price to obtain it during an end-ofseason clearance. We find that enhanced design helps to mitigate strategic behavior by offering consumers a product they value more, making them less willing to risk waiting for a clearance sale and possibly experiencing a stock-out. Quick response mitigates strategic behavior through a different mechanism: by better matching supply to demand, it reduces the chance of a clearance sale. Most importantly, we find that while it is possible for quick response and enhanced design to be either complements or substitutes, the complementarity effect tends to dominate. Hence, when both quick response and enhanced design are combined in a fast fashion system, the firm typically enjoys a greater incremental increase in profit than the sum of the increases resulting from employing either system in isolation, roughly by a factor of two in our numerical experiments. Furthermore, complementarity is strongest when customers are very strategic. We conclude that fast fashion systems can be of significant value, particularly when consumers exhibit strategic behavior.
\end{abstract}

\section{Introduction}

Firms in the fashion apparel industry — such as Zara, H\&M, and Benetton - have increasingly embraced the philosophy of "fast fashion" retailing (Passariello 2008, Rohwedder and Johnson 2008). Generally speaking, a fast fashion system combines at least two components:

1. Short production and distribution leadtimes, enabling a close matching of supply with uncertain demand (which we refer to as quick response techniques). 
2. Highly fashionable ("trendy") product design (which we refer to as enhanced design techniques).

Short leadtimes are enabled through a combination of localized production, sophisticated information systems that facilitate frequent inventory monitoring and replenishment, and expedited distribution methods. For example, Zara, primarily a European retailer, produces the majority of its designs in costly European and North African factories (rather than outsourcing to less expensive Asian facilities), and continuously monitors inventory levels in stores to effectively match supply and demand (Ghemawat and Nueno 2003, Ferdows et al. 2004). The second component (trendy product design) is made possible by carefully monitoring consumer and industry tastes for unexpected fads and reducing design leadtimes. Benetton, for example, employs a network of "trend-spotters" and designers throughout Europe and Asia, and also pays close attention to seasonal fashion shows in Europe (Meichtry 2007). ${ }^{1}$

From an operational perspective, quick response strategies have been relatively well studied, and are known to yield significant value to firms by better matching supply and demand (see, e.g., Fisher and Raman 1996, Eppen and Iyer 1997, Caro and Martínez-de-Albéniz 2009, Caro and Gallien 2009) and by influencing consumer purchasing behavior by reducing the frequency and severity of season-ending clearance sales (Cachon and Swinney 2009). However, the second component of fast fashion systems-creating trendy, highly fashionable products-has received far less attention. Indeed, despite the intense recent interest in leadtime reduction, Meichtry (2007) describes how some firms are attempting to focus on design and develop trendier products without reducing their production leadtimes, due to the difficulties (both logistical and cultural) that can accompany drastically redesigning the supply network.

In this paper, we develop a framework that allows us to address the value of such enhanced design strategies, and subsequently to consider the impact of combining both quick response and enhanced design in a fast fashion system. We postulate that, all else being equal, enhanced design capabilities result in products that are of greater value to consumers and hence elicit a greater willingness-to-pay. Consequently, firms may exploit this greater willingness-to-pay by charging higher prices on "trendy" products than on more conservative products. Enhanced design

\footnotetext{
${ }^{1}$ There are other aspects of fast fashion systems that we do not consider, notably frequent changes in product assortment.
} 
capabilities are costly, however: there are typically fixed costs (a large design staff, trend-spotters, rapid prototyping capabilities, etc.) and there may be greater variable costs (e.g., because of more labor-intensive production processes or costly local labor). Thus, as with any operational strategy, firms considering enhanced design must trade off the benefits of the strategy (greater consumer willingness-to-pay) with the costs (fixed and variable).

A central issue that we address is the impact of enhanced design and quick response on consumer purchasing behavior. Particularly in the fashion apparel industry, the propensity of consumers to anticipate future markdowns and intentionally delay purchasing until a sale occurs is a well documented and widespread problem (Rozhon 2004). This behavior erodes retailer margins and can drastically reduce profitability. Both enhanced design and quick response have frequently been cited as an effective tool for retailers to combat such "strategic" customer behavior (see, e.g., Ghemawat and Nueno 2003). Such systems, we demonstrate, decrease consumer incentives to wait for clearance sales in key two ways. Quick response reduces the chance that inventory will remain to be sold at the clearance price (because quick response more closely matches supply and demandsee Cachon and Swinney 2009). Enhanced product design, on the other hand, gives customers a trendier product that they value more, making them less willing to risk waiting for a sale if there is any chance that the item will stock out. Thus, while quick response decreases the expected future utility of waiting for a price reduction, enhanced design increases the immediate utility of buying the product at the full price. By decreasing consumer incentives to wait for the clearance sale, both enhanced design and quick response allow the firm to set higher selling prices while still inducing consumers to pay the full price.

Because the two techniques are increasingly used in combination in fast fashion systems, a key question is how the two practices interact and influence one another's value; in particular, we consider whether enhanced design and quick response are substitutes (i.e., implementing one practice reduces the marginal worth of the other) or complements (i.e., implementing one practice increases the marginal worth of the other, Milgrom and Roberts 1990). Whether quick response and enhanced design are complements or substitutes has important consequences for the profitability of fast fashion systems versus alternative systems (e.g., a system with only quick response or enhanced design, but not both), and moreover is critical to determine whether the efforts of firms described by Meichtry (2007) to focus on implementing only one aspect of fast fashion are pru- 
dent: as discussed by Milgrom and Roberts (1990), complementary strategies should be adopted simultaneously, whereas substitutable strategies are more likely to be adopted in isolation.

At first glance, it may appear that the answer to the complementarity question is straightforward. Enhanced design results in more consumer value and higher selling prices, so eliminating lost sales becomes more important to the firm with enhanced design (because in each lost sale, the firm will lose out on a higher margin). This implies that adding quick response to an enhanced design system may result in greater incremental value than implementing quick response alone, leading to a complementarity effect.

Our model confirms that this reasoning is correct and, in the absence of strategic consumer behavior, results in quick response and enhanced design being complements. When customers behave strategically, however, we also identify a second, more subtle, substitution effect that arises between quick response and enhanced design. This effect is rooted in the fact that the two practices independently influence consumer purchasing behavior in a similar way. As discussed above, when customers exhibit strategic behavior, both quick response and enhanced design generate value to the firm by reducing consumers incentives to delay a purchase. Because both practices act via similar mechanisms to mitigate strategic customer behavior, they behave can as substitutes along this dimension. If, for instance, adopting quick response alone serves to heavily reduce (or even eliminate) consumer incentives to wait for the clearance sale, then adding enhanced design on top of quick response to form a fast fashion system may have little incremental impact on consumer behavior and hence may reduce the marginal value of enhanced design practices.

As a result of this behavioral substitution effect, quick response and enhanced design can be either complements or substitutes. In the following analysis, we discuss conditions that dictate the direction of this relationship. We find that while substitution is possible-particularly if enhanced design is costly on a marginal basis-under most reasonable conditions the two practices are complements. Thus, when employing both strategies in a fast fashion system, the firm typically enjoys a superadditive increase in profit relative to employing the strategies in isolation. Furthermore, via numerical experiments we show that the complementarity effect is strongest if customers are highly strategic. These results help to demonstrate that, while it may be tempting for firms to only invest in one aspect of fast fashion (either quick response or enhanced design), there is less value in doing so than in pursuing both strategies together-potentially far less value, if consumers 
are highly strategic.

The remainder of this paper is organized as follows. $§ 2$ reviews the relevant literature, while $\S 3$ describes a basic model and analyzes a system with neither quick response nor enhanced design. $\S \S 4-5$ discuss the impact of employing quick response and enhanced design in isolation, and $\S \S 6-7$ consider the combination of both components in a fast fashion system. $\S 8$ reports the results of an extensive numerical study, and $§ 9$ concludes the paper with a discussion of the results.

\section{Literature Review}

There are two primary streams of research that relate to our analysis: the literature on operational flexibility with non-strategic customers (in particular, quick response and postponement practices) and the literature on strategic consumer purchasing behavior. Quick response has received a large amount of attention-see, e.g., Fisher and Raman (1996), Eppen and Iyer (1997), Iyer and Bergen (1997), Fisher et al. (2001), and the Sport Obermeyer case study by Hammond and Raman (1994). Each of these works describes the benefit of reducing supply-demand mismatches by providing the firm with an option to procure inventory after learning updated demand information. More recent works, such as Li and Ha (2008) and Caro and Martínez-de-Albéniz (2009), address the impact of competition on quick response inventory practices. Postponement-the practice of delaying final assembly-also seeks to provide higher product availability with a lower inventory investment; see Lee and Tang (1997), Feitzinger and Lee (1997), Goyal and Netessine (2007), and Anand and Girotra (2007). The distinction between postponement and enhanced design is one of degree. Postponement creates variants from a base model (e.g., different color panels for the same phone) whereas enhanced design creates significantly different product variants from component inventory (e.g., a skirt or dress slacks from the same material). Neither the papers on quick response nor postponement analytically address the impact of quick response or enhanced design on strategic consumer behavior.

The issue of strategic (or rational) customer purchasing behavior dates to Coase (1972) and the theory of durable goods pricing in monopolies. The Coase Conjecture, which was described informally by Coase (1972) and formalized by Stokey (1981) and Bulow (1982), states that in the face of infinitely patient consumers, a monopolist can charge a price no higher than marginal cost, 
as consumers will patiently wait as long as possible for the price to be reduced to its lowest level.

More recently, a stream of research has emerged that explores the role of supply and demand mismatch in influencing strategic consumer purchasing behavior. Liu and van Ryzin (2008) show that a firm may wish to understock to generate shortages when prices decline over time and consumers may strategically wait for the sale. Aviv and Pazgal (2008) examine the value of dynamic and static pricing schemes in a revenue management setting with stochastically arriving strategic customers. Yin et al. (2009) consider the impact of in-store display formats (e.g., displaying all units or displaying one unit to limit consumer information about inventory availability) on the consumer incentive to strategically delay purchasing. Su and Zhang (2008) show that when the sale price is exogenously set, the firm reduces inventory and sets a lower full price in order to induce strategic consumers to purchase at the full price. Other aspects of the strategic consumer purchasing problem that have been addressed include: availability guarantees in Su and Zhang (2009), product returns in $\mathrm{Su}$ (2009), and consumer stockpiling in $\mathrm{Su}$ (2007). While many of these papers consider the inventory decision of the firm, none address the interaction of quick response, enhanced design, or fast fashion systems with consumer purchasing.

Cachon and Swinney (2009) and Swinney (2009) do address the impact of quick response on strategic consumer purchasing. Cachon and Swinney (2009) show that the presence of strategic consumers can enhance the value of quick response beyond just matching supply with demand adopting quick response reduces the likelihood of deep discounts, which makes strategic consumers more willing to purchase at the regular price. In Swinney (2009), the impact of quick response in markets where consumers learn about product value over time is explored, and it is shown that quick response may decrease or increase the firm's profit, depending on characteristics of the selling environment (e.g., whether consumer returns are allowed or whether the firm prices dynamically). Unlike the present analysis, these papers do not address the impact of enhanced design on consumer purchasing behavior nor the interaction between enhanced design and quick response to generate a fast fashion retail system. 


\begin{tabular}{l|ll} 
& Normal Design & Enhanced Design \\
\hline Slow Production & Traditional $(T)$ & Enhanced Design $(D)$ \\
Quick Response & Quick Response $(Q)$ & Fast Fashion $(F)$
\end{tabular}

Table 1. The four possible production systems.

\section{The Traditional System}

To stimulate our analysis of the incremental value of the components of a fast fashion system, we analyze a total of four potential operational systems. A traditional system, abbreviated $T$, represents a typical retailer with long production leadtimes and standard product design abilities. As we will reveal below, this system most closely resembles a newsvendor model. A quick response system, abbreviated $Q$, does not employ enhanced design capabilities, but does yield significantly reduced production leadtimes. An enhanced design system, abbreviated $D$, employs enhanced design capabilities (and hence greater consumer willingness-to-pay) but maintains long production leadtimes-this system resembles the efforts described by Meichtry (2007) to focus on product design while avoiding the kind of radical supply chain overall necessary to achieve leadtime reduction. Finally, a fast fashion system, abbreviated $F$, employs both quick response and enhanced design capabilities. The fast fashion system resembles the mode of operations increasingly found in retailers such as Zara, Benetton, and H\&M. The characteristics of these systems are summarized in Table 1.

One could argue that short production leadtimes should increase the efficacy of creating trendy products by allowing designs to be finalized closer to the selling season. For example, many traditional fashion retailers (such as Gap) have average design and production leadtimes on the order of six to twelve months. If these firms intensified their product design efforts without reducing production leadtimes, while they may be able to generate better products overall, they would still have to make final design decisions months in advance of the selling season (and consequently well in advance of the revelation of any unexpected trends). On the other hand, a fast fashion firm has dramatically shorter design-to-shelf leadtimes - in some cases, on the order of weeks - and so such firms can observe and replicate trends practically in real time. Thus, enhanced design efforts presumably result in an even greater increase in consumer willingness-to-pay if the firm simultaneously achieves leadtime reduction. We take a conservative approach on this issue: we assume that 
adopting enhanced design capabilities results in an identical increase in consumer willingness-to-pay regardless of the production leadtime of the firm. In other words, we do not assume, ex ante, that any complementarity exists between enhanced design efforts and quick response capabilities-we discuss the impact of this assumption in the conclusion of the paper.

In each possible system depicted in Table 1, we analyze a game between a firm and its consumers. The firm chooses the selling price and the inventory level, while consumers choose whether to buy at the full price or wait for a potential clearance sale (running the risk that the product might run out). In this section, we introduce the basic model and analyze the case of the traditional system-that is, a system possessing neither quick response nor enhanced design. This model will serve as a base case, upon which we will expand to analyze the three alternative systems.

\subsection{The Model}

A single firm sells a single product over a finite season. The market is characterized by demand uncertainty: the total number of consumers in the market is stochastic and denoted by the continuous random variable $N$ with distribution $F(\cdot)$ and mean $\mu$. Consumers have homogenous value $v$ for the product.

The product is sold over a single season. Prior to the start of the selling season (and prior to learning market size), the firm makes an inventory procurement $q$ at unit cost $c$, and sets a selling price, $p$, to maximize expected profit, $\pi(q, p)$. At the end of the season, all remaining inventory is cleared at an exogenous salvage or "sale" price $s$, where $s<c{ }^{2}$

Customers are strategic to the extent that they are forward-looking: they recognize that the product will eventually be reduced in price and consider delaying their purchase until the price is lowered. Customers discount future consumption at a rate $\delta \in[0,1]$. By delaying a purchase until the clearance sale, customers lose out on some consumptive value, and hence their future utility is reduced to reflect this loss. In addition, $\delta$ may be thought of as the level of strategic behavior or patience of the customer population (higher $\delta$ implies more patient or strategic consumers) or as a proxy for the durability of the good (higher $\delta$ implies a more durable good with greater

\footnotetext{
${ }^{2} \mathrm{Su}$ and Zhang (2008) also assume that the clearance price $s$ is exogenous and common knowledge (e.g., it may be the customary sale price in the industry or for the firm, or it may be the prevailing price of a secondary salvage market that is accessible to consumers as well as the firm). An alternative model would allow the firm to dynamically set a sale price at the end of the regular season; for a model with heterogeneous customers and dynamic sale pricing coupled with quick response, see Cachon and Swinney (2009).
} 
future value). For the remainder of the paper, we adopt the convention that greater $\delta$ implies a "more strategic customer population," with the understanding that the factors influencing this may be related to the product itself, overall market or industry conditions, or intrinsic consumer characteristics.

All consumers arrive at the firm at the start of the selling season. After observing the selling price $p$, each consumer individually chooses to either purchase the product immediately at price $p$ or delay her purchase until the clearance sale. When making this decision, consumers take into account their surplus from an immediate purchase (a function of valuation and price) and their expected surplus from a delayed purchase, which incorporates the clearance price $s$, the discount factor $\delta$, and the perceived probability of obtaining a unit, which we label $\phi$. One of two cases then occurs for each individual consumer. If the firm is out-of-stock at the full price, the game is over. If the firm is in-stock, then the consumer chooses between purchasing at the full price and obtaining the unit for certain, and delaying until the clearance sale and probabilistically obtaining a unit. The surplus of an immediate purchase at price $p$ is $v-p$, while the expected surplus of a delayed purchase at the clearance price $^{3}$ is $\delta \phi(v-s)$. Consumers subsequently choose to purchase at the price that yields greater expected surplus, and we assume that if consumers are indifferent between the two actions, then they purchase at the full price $p .{ }^{4}$ The sequence of events is depicted in Figure 1.

Strategic consumers who choose to delay their purchase are "first in line" in the clearance market-that is, while the firm may dispose of an infinite amount of inventory on the salvage market (implying infinite demand), strategic customers are allocated remaining inventory first, followed by demand from the salvage market. ${ }^{5}$ In what follows, we use the superscript $*$ to denote equilibrium

\footnotetext{
${ }^{3}$ An alternative model would be consumers who do not discount future consumption, but rather have declining valuations. In that case, the expected surplus of a delayed purchase at the clearance price is $\delta v-s$; see, e.g., Cachon and Swinney (2009). This alternative model results in slightly higher full prices (because consumers consider the full future cost, $s$, rather than a discounted future cost, $\delta s$ ) but qualitatively similar results to our own.

${ }^{4}$ In particular, if consumers are indifferent between purchasing opportunities, they do not consider randomizing between the two periods; in other words, we do not consider mixed strategies. The reason for this is simple: because our consumers are homogenous, if mixed strategies are allowed and some consumers (randomly) choose to wait for the sale, the firm can simply lower the full price by an arbitrarily small amount to eliminate consumer indifference and induce all consumers to pay the full price. The amount of discounting necessary to achieve this is arbitrarily small and is hence ignored.

${ }^{5}$ This allocation rule is also adopted by Su and Zhang (2008). A more general allocation mechanism in the salvage stage-e.g., random arrivals of strategic customers and customers from the exogeneous salvage market, discussed in Cachon and Swinney (2009)-merely reduces the probability that a consumer receives a unit at the salvage price and is unlikely to qualitatively change the results.
} 


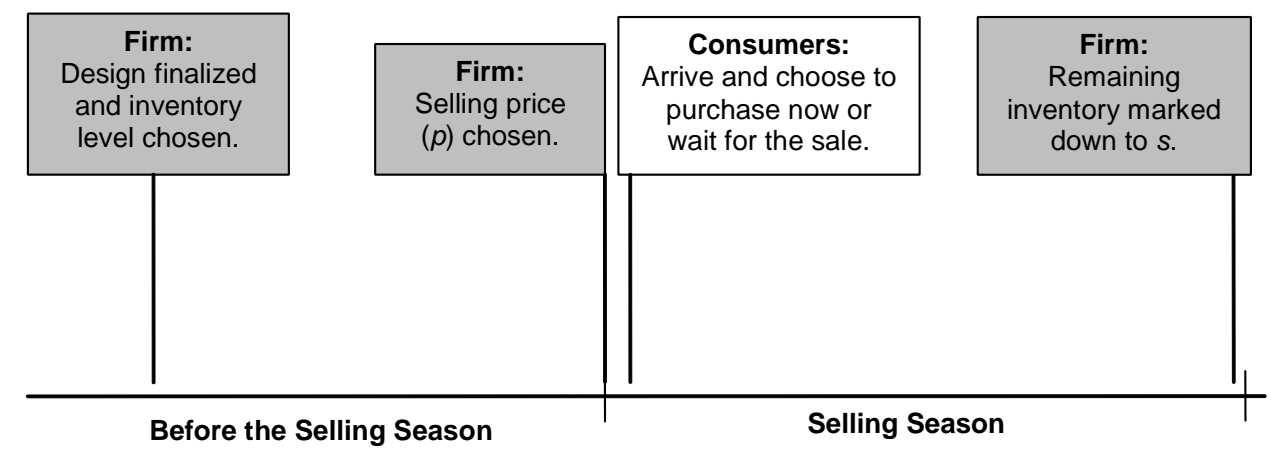

Figure 1. Sequence of events in the traditional system.

values (prices, quantities, profits), and the subscripts $T, Q, D$, and $F$ to denote specific systems where necessary.

Finally, we note here that we do not consider any fixed costs resulting from the implementation of any system (though we will account for increases in variable costs resulting from quick response or enhanced design). Indeed, fixed costs can be significant, particularly in the form of physical infrastructure (factory, warehouse, and distribution systems) and information systems. Directionally, the impact of such fixed costs is clear.

\subsection{Equilibrium Inventory and Pricing}

To explore the value of the traditional system (and each of the subsequent systems), we analyze a game between the forward-looking customer population and the firm: consumers choose when to buy the product (at the full price or at the discounted price) and the firm chooses how much inventory to stock and what price to charge. We assume that consumers do not directly observe the total inventory of the firm before making their decisions, ${ }^{6}$ and consequently the firm cannot credibly convey inventory information to consumers (i.e., the firm is not a leader in a sequential game). Consumers do, however, make their purchasing decisions with a fixed belief about the probability of a clearance sale $(\phi)$ which is correct in equilibrium-in other words, consumers have rational expectations concerning the average probability of a clearance sale.

We thus seek Nash equilibria in a simultaneous decision game between many players: the firm

\footnotetext{
${ }^{6}$ Consumers may be incapable of directly observing inventory in a variety of situations, including: if the firm is an online retailer; if the firm stocks a particular retail location from a centralized warehouse; or if the firm displays a limited amount of inventory on the store floor.
} 
and a continuum of (identical) consumers. Given that consumers are homogeneous, either all consumers purchase at price $p$ or all consumers purchase at price $s$. However, the latter does not lead to an interesting equilibrium: given $s<c$, the firm does not order any inventory. Thus, we are left to derive an equilibrium in which all consumers purchase early. In such an equilibrium, the firm's expected profit as a function of the price $p$ and quantity $q$ is

$$
\pi_{T}(q, p)=\mathbb{E}((p-s) S(q)-(c-s) q)
$$

where $S(q)=\mathbb{E} \min (q, N)$ is expected sales given a quantity $x$ and the expectation operator $\mathbb{E}$ is taken over market size, $N$. Given these preliminaries, we may now define the equilibrium to pricing-inventory-purchasing game:

Definition 1 An equilibrium with rational expectations to the game between strategic consumers and the firm satisfies:

1. The firm sets price and inventory to maximize expected profit, given that consumers all purchase early: $\left(q^{*}, p^{*}\right)=\arg \max _{q^{*}, p^{*}} \pi(q, p)$.

2. Consumers purchase early, given the selling price $p^{*}$ and a belief about the probability of a clearance sale, $\phi: v-p^{*} \geq \delta \phi(v-s)$.

3. Consumer beliefs are rational: $\phi=F\left(q^{*}\right)$.

Our model of the traditional system is similar to the model analyzed by Su and Zhang (2008), but our consumers discount future consumption by an arbitrary amount. This difference results in slightly more complicated expressions for equilibrium price and inventory levels, but nevertheless the equilibrium analysis is qualitatively similar to our own. Define

$$
A(v)=v(1-\delta)+(1+\delta) s \text { and } B(v, c)=s v-\delta c(v-s)
$$

We may now solve for the equilibrium in the traditional system:

Lemma 1 In a traditional system, an equilibrium with non-zero production exists and is unique. 
In equilibrium, all consumers purchase early. The equilibrium full price is

$$
p_{T}^{*}=\frac{A(v)+\sqrt{A(v)^{2}-4 B(v, c)}}{2} .
$$

Proof. All proofs appear in the appendix.

It is clear that the equilibrium price $p_{T}^{*}$ is decreasing in the consumer discount factor $(\delta)$, hence the greater the severity of strategic customer behavior (i.e., the less consumers discount future consumption and the greater $\delta$ ), the lower the firm must set the selling price to induce consumers to purchase at the full price.

\section{Quick Response}

In the quick response system, the design abilities are standard while the production phase is fasthence, while the product design process results in lower value products for consumers, the inventory may be procured after learning total market size. To model quick response, we adopt a stylized model employed by much of the literature; see, e.g., Cachon and Swinney (2009), Fisher and Raman (1996), and Eppen and Iyer (1997). Following this literature, we assume that the firm can procure inventory both before and after receiving a forecast update prior to the start of the selling season. The forecast update is perfectly informative (i.e., reveals the actual demand level) and production is fast enough that all units arrive before the start of the selling season. Inventory procured prior to learning demand information is obtained for a low cost (c, just as in the traditional system in the preceding section), while additional inventory procured after learning the realized value of market size incurs an additional cost $c_{Q} \geq 0$ due to expedited manufacturing and shipping expenses. The sequence of events is depicted in Figure 2.

When making the inventory procurement following the realization of demand information, it is easy to see that as long as the margin on each unit $\left(p-c-c_{Q}\right)$ is positive, the optimal action of the firm is to produce precisely enough inventory to cover all full price demand. Equilibrium in the quick response system is defined using the same three conditions in Definition 1, and the following lemma solves for this equilibrium.

Lemma 2 In a quick response system, an equilibrium with non-zero production exists and is unique. 


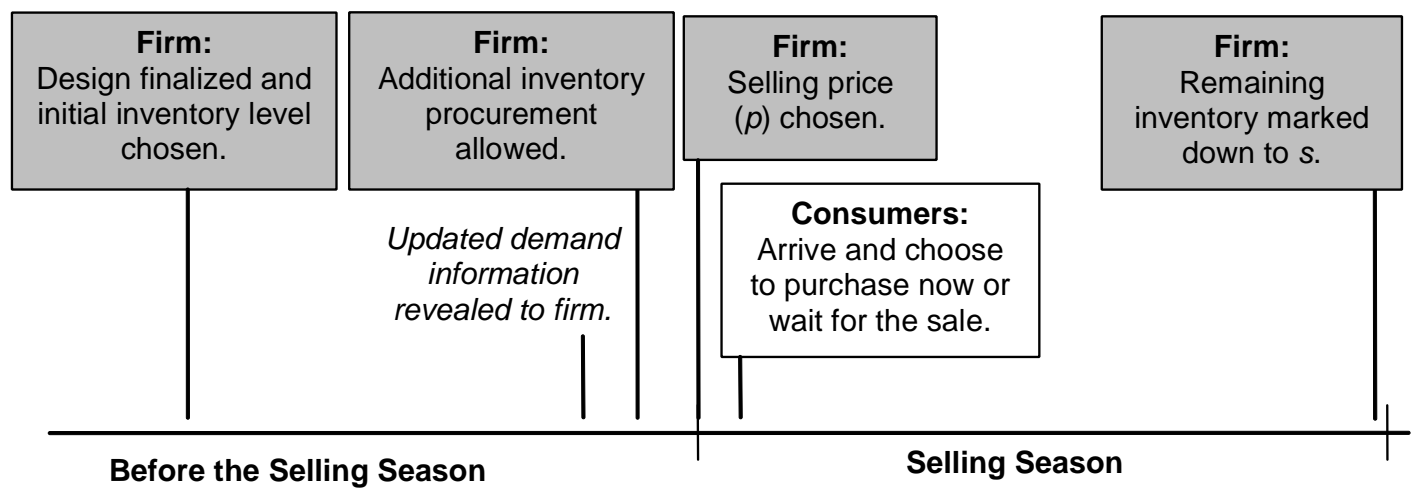

Figure 2. Sequence of events in the quick response system.

In equilibrium, all consumers purchase early. The equilibrium full price is

$$
p_{Q}^{*}=v-\delta \frac{c_{Q}}{c+c_{Q}-s}(v-s)
$$

if $p_{Q}^{*} \geq c+c_{Q}$, while if $p_{Q}^{*}<c+c_{Q}$, the equilibrium is identical to the traditional system.

Due to the option to procure additional inventory at a later date, the firm procures less inventory in the initial buy than in the traditional system, which results in a lower chance that there will be inventory available during the clearance season. Consequently, from a consumer's point of view, the probability of successfully obtaining a unit at the sale price decreases, along with the incentive to wait for the discounted price. In turn, this allows the firm to charge a higher full price while maintaining an equilibrium in which (as we saw in the traditional system) all consumers attempt to purchase at the full price, provided the extra cost of quick response $\left(c_{Q}\right)$ is not too high, as the following lemma summarizes:

Lemma 3 The equilibrium price is greater in the quick response system than in the traditional system $\left(p_{Q}^{*}>p_{T}^{*}\right)$ if and only if $p_{T}^{*}>c+c_{Q}$.

In sum, quick response provides value to the firm via two distinct effects:

1. The sales effect: quick response eliminates lost sales by allowing the firm a second procurement opportunity after observing demand. 
2. The behavioral effect: as Lemma 3 demonstrates, when the cost of quick response is not too high, value is also added by reducing consumer incentives to wait for the sale and subsequently increasing the equilibrium selling price. ${ }^{7}$

One may think of the sales effect as the operational consequence of quick response (well studied in the literature, e.g., by Fisher and Raman 1996) while the latter effect is purely a consequence of strategic customer behavior. The fact that quick response generates value via two independent mechanisms is critical when we discuss the value of fast fashion in $\S 7$.

\section{Enhanced Design}

In the enhanced design system, the production leadtimes are long but the firm invests in improved design efforts that result in greater value to consumers. Thus, we assume that enhanced design results in a marginal increase of $m \geq 0$ to consumer value, that is, consumers possess valuations equal to $v+m$ for products resulting from enhanced design efforts. ${ }^{8}$ However, when operating with enhanced design capabilities, every unit produced incurs an additional cost $c_{D} \geq 0$. To facilitate our analysis, the clearance price $s$ is assumed to be identical to the clearance price in the traditional and quick response systems. ${ }^{9}$ The sequence of events is identical to that depicted in Figure 1.

Due to the similarity in the sequence of events, the analysis of the enhanced design system is comparable to that of the traditional system. Thus, the following lemma follows immediately from Lemma 1.

Lemma 4 In a enhanced design system, an equilibrium with non-zero production exists and is unique. In equilibrium, all consumers purchase early. The equilibrium full price is

$$
p_{D}^{*}=\frac{A(v+m)+\sqrt{A(v+m)^{2}-4 B\left(v+m, c+c_{D}\right)}}{2} .
$$

\footnotetext{
${ }^{7}$ In Cachon and Swinney (2009), quick response provides value by influencing the firm's dynamic sale pricing decisions during the selling season; here, the sale price is exogenously fixed, and quick response provides value by influencing the firm's initial pricing decision at the start of the season.

${ }^{8}$ In our model, enhanced design results in greater consumer value, which the firm then exploits to raise the selling price. An alternative model might assume that the selling price is fixed (possibly for competitive reasons), but enhanced design results in a more popular product and hence greater market share or size. Such a model, particularly one incorporating competition, may prove to be a fruitful direction for future research.

${ }^{9}$ One might (justifiably) argue that the clearance price should be higher in a system with enhanced design. This turns out to significantly complicate the analytical price and profit comparisons in our model; hence, we numerically investigate this possibility in $\S 8.3$.
} 
Note that $p_{D}^{*}$ is increasing in $m$ and $c_{D}$ while the behavior of $p_{D}^{*}$ as a function of the other parameters is identical to the behavior of $p_{T}^{*}$. Hence, because the traditional system is equivalent to the enhanced design system with $m=c_{D}=0$, it follows that $p_{D}^{*}>p_{T}^{*}$, which we formally state in the following lemma:

Lemma 5 The equilibrium price is greater in the enhanced design system than in the traditional $\operatorname{system}\left(p_{D}^{*}>p_{T}^{*}\right)$.

Although the price is higher with the enhanced design system, the equilibrium consumer action remains the same as the traditional system: all customers purchase at the full price rather than wait for the sale. Thus, the firm can exploit enhanced design capabilities to raise prices without increasing strategic waiting, which is clearly beneficial to the firm if the increase in costs $\left(c_{D}\right)$ is not too high. A necessary condition for enhanced design to be profitable is $p_{T}^{*}<p_{D}^{*}-c_{D}$, which implies that the margin on each sale increases as a result of enhanced design. Note that this is not a sufficient condition for the profitability of enhanced design, as an increase in production costs also implies an increase in costs due to excess inventory.

The preceding lemmas demonstrate that enhanced design, much like quick response, influences firm profit via two distinct effects:

1. The valuation effect: adding $m$ to valuations raises the utility that customers earn from the product whether they buy it at the full price or the clearance price; however, since $\delta$ and $\phi$ are both less than one, utility from early purchasing is raised more than utility from late consumption $(m>\delta \phi m)$, allowing the firm to raise the full price and still induce early purchasing.

2. The behavioral effect: by changing consumer valuations and the production cost, the firm alters costs of holding too much and too little inventory, in turn changing the optimal inventory level and hence the probability of a clearance sale. Because optimal inventory may increase or decrease relative to the traditional system, consumers may have more or less incentive to strategically wait for the sale, and the behavioral effect may have negative or positive value to the firm. 
Just as in the quick response case, the first mechanism (the valuation effect) exists even if customers are completely non-strategic; the latter effect, on the other hand, only exists if customers exhibit strategic behavior. Unlike the quick response case, both of these effects impact profit by affecting the equilibrium selling price.

\section{Fast Fashion}

The fast fashion system combines operating characteristics of the quick response and enhanced design systems. As a result, the firm is capable of both raising consumer values for the product and reducing supply-demand mismatch. The sequence of events in the fast fashion system is the same as that depicted in Figure 2. As in the enhanced design model, consumers earn an extra value of $m$ per unit, and every unit incurs an additional cost of $c_{D} \geq 0$. As in the quick response system, the firm has the option of obtaining additional inventory close to the selling season after receiving perfect demand information, at an additional cost of $c_{Q} \geq 0$ per unit. Thus, the firm possesses a comparable cost structure to the alternative systems.

Because the sequence of events is similar in the quick response and the fast fashion systems, the equilibrium follows immediately from Lemma 2 by setting consumer valuations equal to $v+m$ and increasing the production cost on every unit (procured both before and after the forecast update) by $c_{D}$.

Lemma 6 In a fast fashion system, an equilibrium with non-zero production exists and is unique. In equilibrium, all consumers purchase early. The equilibrium full price is

$$
p_{F}^{*}=v+m-\delta \frac{c_{Q}}{c+c_{D}+c_{Q}-s}(v+m-s)
$$

Using Lemma 6, we derive the following result:

Lemma 7 The equilibrium price is greater in the fast fashion system than in all of the other systems $\left(p_{F}^{*}>\max \left(p_{D}^{*}, p_{Q}^{*}, p_{T}^{*}\right)\right)$ if $p_{D}^{*}>c+c_{D}+c_{Q}$.

In other words, the firm can leverage a fast fashion system to raise the equilibrium selling price in multiple ways via the mechanisms generated by the component strategies of fast fashion: quick 
response allows the firm to raise the price via the behavioral effect, while enhanced design allows the firm to alter the selling price via both the valuation and behavioral price effects. The combination of these effects results in a fast fashion system yielding the greatest equilibrium price (provided, as in the quick response system, costs are not too high so as to make the second inventory procurement option unprofitable).

Although Lemma 7 demonstrates that fast fashion results in higher equilibrium selling prices, this does not necessarily imply that a fast fashion firm (such as Zara) will have greater prices than a firm using traditional production. Indeed, Zara famously has lower initial selling prices than many of its rivals. This apparent discrepancy is due to the fact that our analysis compares prices for different production systems holding all else equal; in particular, baseline product quality. In addition to being famous for low prices and fast fashion production, Zara is also known to use cheaper materials, resulting in less durable, lower quality products (designed to "be worn 10 times," as Ghemawat and Nueno 2003 note). Hence, for Zara, $v$ (base consumer value) and $c$ (base production cost) are both likely to be lower than at a higher quality competitor, such as a traditional department store, resulting in lower prices at Zara despite the implementation of fast fashion production.

\section{The Interaction of Enhanced Design and Quick Response}

In this section, we analyze the impact of combining enhanced design and quick response in a fast fashion system. Specifically, we seek to answer the following question: are enhanced design and quick response complements, or substitutes? If they are complements, then investing in a fast fashion system results in a superadditive benefit: the incremental value of a fast fashion system (the change in profit over a traditional system) is more than the combined incremental value of enhanced design and quick response employed in isolation, i.e.,

$$
\left(\pi_{F}^{*}-\pi_{T}^{*}\right)>\left(\pi_{Q}^{*}-\pi_{T}^{*}\right)+\left(\pi_{D}^{*}-\pi_{T}^{*}\right)
$$

Simplifying this expression, quick response and enhanced design are complements if and only if $\pi_{F}^{*}-\pi_{Q}^{*}>\pi_{D}^{*}-\pi_{T}^{*}$ 


\begin{tabular}{|c|c|c|c|}
\hline & Quick Response & Interaction & Enhanced Design \\
\hline $\begin{array}{l}\frac{\pi}{0} \\
\frac{0}{0} \\
\frac{0}{0} \\
\frac{0}{0} \\
0\end{array}$ & $\begin{array}{c}\text { Sales Effect } \\
\text { Eliminate lost sales. }\end{array}$ & Complements & $\begin{array}{c}\text { Valuation Effect } \\
\text { Add } m \text { to consumer } \\
\text { valuations and raise the price. }\end{array}$ \\
\hline 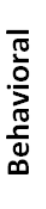 & $\begin{array}{l}\text { Behavioral Effect } \\
\text { Lower the chance of a } \\
\text { clearance sale, } \\
\text { leading to a greater } \\
\text { equilibrium price. }\end{array}$ & $\begin{array}{l}\text { Complements } \\
\text { or } \\
\text { Substitutes }\end{array}$ & $\begin{array}{l}\text { Behavioral Effect } \\
\text { Alter (raise/lower) chance of } \\
\text { a clearance sale, leading to a } \\
\text { different (lower/higher) } \\
\text { equilibrium price. }\end{array}$ \\
\hline
\end{tabular}

Figure 3. The key interactions between quick response and enhanced design.

Our first result is that, in general, it is possible for quick response and enhanced design to be either complements or substitutes. To explain this finding, recall that quick response impacts profit via a sales effect (eliminating lost sales) and a behavioral effect (influencing consumer purchasing behavior, allowing for a greater selling price). Enhanced design impacts profit via a valuation effect (adding $m$ to consumer valuations) and a behavioral effect (altering consumer incentives to strategically wait for the sale). There are two key interactions at work: the interaction of the sales effect of quick response and the valuation effect of enhanced design, which we call the operational interaction; and the interaction of the behavioral effects of both practices, which we call the behavioral interaction. These terms derive from the fact that the operational interaction exists for any $\delta$ (including $\delta=0$, though we emphasize that the interaction is not independent of $\delta$ ), whereas the behavioral interaction only exists if $\delta>0$, i.e., if consumers are strategic. The interactions are summarized in Figure 3. ${ }^{10}$

As shown in Figure 3, the key to our initial result lies in the fact that while the operational interaction is unambiguously complementary, along the behavioral dimension quick response and enhanced design may be either complements or substitutes. The net effect is the sum of the operational and behavioral interactions, hence when the behavioral substitution effect is sufficiently strong, quick response and enhanced design may, overall, be substitutes.

\footnotetext{
${ }^{10}$ We have focused on the two most salient effects which lead to complementarity or substitution. However, there are other interactions involved in a fast fashion system beyond these two: for instance, the behavioral effect of quick response interacts with the valuation effect of enhanced design. Moreover, increased marginal cost in the enhanced design system also impacts supply-demand mismatch costs, though as these effects are more standard we have focused on the behavioral consequences of enhanced design.
} 
To discuss this result, we will analyze each interaction separately, beginning with the operational interaction. As stated above, quick response and enhanced design are always operational complements. This is because increasing consumer valuations and thus the selling price (adopting enhanced design) is more valuable to the firm if sales are higher (i.e., if the firm also employs quick response) and the marginal increase in price is earned on more units. To illustrate this point, consider the case when $\delta=0, s=0$, and $c_{Q}=c_{D}=0$. Imposing $\delta=0$ eliminates the behavioral effects from Figure 3, meaning only the operational interaction remains. From the preceding lemmas, the optimal selling prices are $p_{T}^{*}=p_{Q}^{*}=v$ and $p_{D}^{*}=p_{F}^{*}=v+m$. The incremental change in expected profit from enhanced design is

$$
\begin{aligned}
\pi_{D}^{*}-\pi_{T}^{*} & =(v+m) S\left(q_{D}^{*}\right)-c q_{D}^{*}-v S\left(q_{T}^{*}\right)+c q_{T}^{*} \\
& \leq(v+m) S\left(q_{D}^{*}\right)-c q_{D}^{*}-v S\left(q_{D}^{*}\right)+c q_{D}^{*} \\
& =m S\left(q_{D}^{*}\right) \leq m \mu=\pi_{F}^{*}-\pi_{Q}^{*} .
\end{aligned}
$$

The inequality follows from the fact that, in the traditional system, profit evaluated at quantity $q_{D}^{*}$ is less than profit evaluated at quantity $q_{T}^{*}$, by definition of the optimal quantity $q_{T}^{*}$. As the example shows, with fast fashion, the additional margin from enhanced design is enjoyed on the mean demand, while with enhanced design (and no quick response abilities) the additional margin is only enjoyed on the expected sales (mean demand minus lost sales), which are by definition less than the mean demand. Consequently, enhanced design's valuation effect is more beneficial if the firm also possesses quick response, leading to a complementary relationship.

If the operational interaction were the only interaction between quick response and enhanced design, then the two practices would always be complements, and indeed this is the case if $\delta=0$ (i.e., if consumers are non-strategic). However, when $\delta>0$, the behavioral effects of these strategies come into play, potentially leading to substitution. To understand this behavioral interaction, it is useful to consider two specific examples, one in which quick response and enhanced design are complements along the behavioral dimension and one in which they are substitutes. In both examples, demand is normally distributed with $\mu=150$ and $\sigma=75$, and $\delta=0.9, v=8, c=2$, $s=1.9, c_{Q}=0$, and $m=1$.

Example 1: Behavioral Complementarity. In the first example, $c_{D}=0$. In the traditional 
and enhanced design systems, equilibrium prices are $p_{T}^{*}=3.44$ and $p_{D}^{*}=3.65$. Expected profits in these systems are $\pi_{T}^{*}=201$ and $\pi_{D}^{*}=232$, and the incremental value of enhanced design is $\pi_{D}^{*}-\pi_{T}^{*}=31$. In the quick response and fast fashion systems, equilibrium prices are $p_{Q}^{*}=8$ and $p_{F}^{*}=9$ (costless quick response means the firm produces all inventory after learning demand, allowing the firm to eliminate clearance sales and extract all consumer surplus), with expected profits equal to $\pi_{Q}^{*}=\left(p_{Q}^{*}-c\right) \mu=900$ and $\pi_{F}^{*}=\left(p_{F}^{*}-c-c_{D}\right) \mu=1050$. The incremental value of a fast fashion system over a quick response system is $\pi_{F}^{*}-\pi_{Q}^{*}=150$, and so in this example, quick response and enhanced design are complements.

The reason for this is that, in addition to the operational complementarities, quick response and enhanced design are complements along the behavioral dimension as well. This can be seen in the increase in the equilibrium price resulting from enhanced design. Adding enhanced design to a traditional system only results in a price increase of $p_{D}^{*}-p_{T}^{*}=0.21$, while adding enhanced design to quick response to form a fast fashion system yields a price increase of $p_{F}^{*}-p_{Q}^{*}=1$. Thus, in this example, enhanced design results in a larger increase in the equilibrium price when used in conjunction with quick response.

Example 2: Behavioral Substitution. In the second example, $c_{D}=m=1$. In the traditional and enhanced design systems, equilibrium prices are $p_{T}^{*}=3.44$ and $p_{D}^{*}=5.21$. Expected profits are $\pi_{T}^{*}=201$ and $\pi_{D}^{*}=241$, hence the incremental value of enhanced design is $\pi_{D}^{*}-\pi_{T}^{*}=41$. In the quick response and fast fashion systems, equilibrium prices remain $p_{Q}^{*}=8$ and $p_{F}^{*}=9$, but expected profits are equal to $\pi_{Q}^{*}=\left(p_{Q}^{*}-c\right) \mu=900$ and $\pi_{F}^{*}=\left(p_{F}^{*}-c-c_{D}\right) \mu=900$. The incremental value of fast fashion over quick response is thus $\pi_{F}^{*}-\pi_{Q}^{*}=0$, clearly less than the value of enhanced design in isolation; hence, quick response and enhanced design are substitutes. Observe that in this example $p_{D}^{*}-p_{T}^{*}=1.77>1=p_{F}^{*}-p_{Q}^{*}$. That is, enhanced design results in a larger increase in the equilibrium price when used in isolation than when used in conjunction with quick response. This is the driving force behind the substitution effect.

The key to both of these examples, and to the behavioral interaction, lies in the way that enhanced design impacts equilibrium inventory. Recall that in all four systems, all consumers attempt to purchase at the full price-hence, the only differences in inventory derive from differences in the costs of ordering too little and too much inventory in the initial procurement. Table ?? summarizes these costs, in addition to the newsvendor critical ratio, for each of the four systems. 


\begin{tabular}{l|cccc}
\hline \hline & $T$ & $D$ & $Q$ & $F$ \\
\hline Underage Cost & $p_{T}^{*}-c$ & $p_{D}^{*}-c-c_{D}$ & $c_{Q}$ & $c_{Q}$ \\
Overage Cost & $c-s$ & $c+c_{D}-s$ & $c-s$ & $c+c_{D}-s$ \\
Critical Ratio & $\frac{p_{T}^{*}-c}{p_{T}^{*}-s}$ & $\frac{p_{D}^{*}-c-c_{D}}{p_{D}^{*}-s}$ & $\frac{c_{Q}}{c+c_{Q}-s}$ & $\frac{c_{Q}}{c+c_{Q}+c_{D}-s}$ \\
\hline \hline
\end{tabular}

Table 2. Equilibrium overage costs, underage costs, and critical ratios.

The behavioral effect of quick response derives from the change in underage costs, while the behavioral effect of enhanced design derives from the change in overage and underage costs. In both cases, changes in costs change the inventory level of the firm and consumer incentives to delay purchasing. If adopting enhanced design increases the critical ratio, then consumers are given a greater incentive to delay purchasing, meaning the firm must lower the selling price somewhat to induce early purchases; in this case, the behavioral effect reduces firm profit. If adopting enhanced design decreases the critical ratio, then consumers have lower incentives to delay purchasing as a result of enhanced design, and hence the behavioral effect increases firm profit.

From Table ??, the critical ratio in the fast fashion system is lower than in the quick response system; hence, enhanced design's behavioral effect always increases firm profit if used in conjunction with quick response. On the other hand, the critical ratio in the enhanced design system may be smaller or larger than the critical ratio in the traditional system; consequently, when used in isolation, the behavioral effect of enhanced design may have positive of negative value to the firm.

In Example 1, quick response and enhanced design are complements because, since $c_{D}=0$, from Lemma 5 (which shows $p_{D}^{*}>p_{T}^{*}$ ), underage costs are higher in the enhanced design system than in the traditional system, while overage costs are the same, meaning the critical ratio is higher with enhanced design than without. The behavioral effect of enhanced design thus has negative value to the firm. The behavioral effect of quick resposne completely eliminates consumer incentives to wait for the sale in both the $Q$ and $F$ system (since $c_{Q}=0$ ), which implies that employing quick response at the same time takes a negative effect and eliminates it, leading to complementarity.

In Example 2, since $c_{D}=1$, the critical ratio is lower with enhanced design than in the traditional system. Consequently, the behavioral effect of enhanced design has positive value to the firm. Once again, because $c_{Q}=0$, the behavioral effect of quick response eliminates the 
behavioral effect of enhanced design, so there is no behavioral benefit to adding enhanced design to quick response system. Thus, quick response takes a positive effect of enhanced design and eliminates it, leading to a substitution effect.

Generally speaking, the behavioral effect of quick response reduces the impact of the behavioral effect of enhanced design. The key to the net interaction of the two practices-whether they are complements or substitutes-lies in whether the behavioral effect of enhanced design has positive or negative value to the firm, which naturally depends on specific parameter values. We may, however, make a definitive statement about the interaction of enhanced design and quick response when $c_{D}=0$ :

Theorem 1 If $c_{D}=0$ and $c_{Q}<s+\sqrt{(v+m-s)(c-s)}-c$, enhanced design and quick response are complements.

The second condition in Theorem 1 ensures that quick response is not so costly that it is unprofitable-the condition guarantees that units procured using quick response have a positive margin, otherwise the firm would not employ quick response. As a result, this is not a particularly restrictive condition. The first condition $\left(c_{D}=0\right)$ is more substantive, ensuring that enhanced design results in no additional marginal production cost, which, in accordance with Example 1 above, implies that enhanced design's behavioral effect is detrimental to the firm.

Based on this discussion, as one might expect, for small $c_{D}$ the behavioral effects of quick response and enhanced design are complements (because the behavioral effect of enhanced design has negative value) while for large $c_{D}$ they are substitutes (because the behavioral effect of enhanced design has positive value, and the behavioral effect of quick response reduces the impact of this effect). We have observed that this is indeed the case, and moreover the substitution effect typically grows stronger as $c_{D}$ increases, a feature that is graphically depicted in Figure 4 for the same parameter combination used in the preceding examples.

While we do not analytically prove the behavior depicted in the figure, we have observed that substitution occurs above some threshold $c_{D}$ in all numerical cases we have examined, and it can be shown for the special case of $c_{Q}=0$ and $\delta=1$ that this occurs (proof omitted).

To summarize, the behavioral effects of the two strategies both serve to independently influence consumer purchasing incentives, and the behavioral effect of quick response always reduces the 


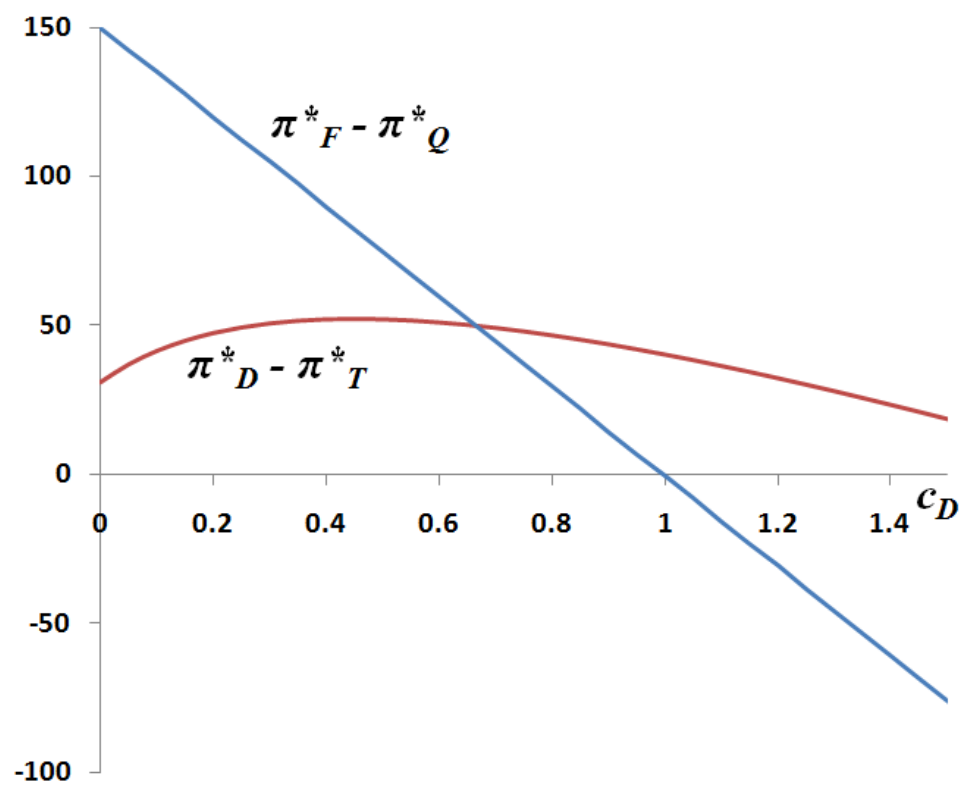

Figure 4. The incremental value of fast fashion over quick response $\left(\pi_{F}^{*}-\pi_{Q}^{*}\right)$ and the incremental value of enhanced design over the traditional system $\left(\pi_{D}^{*}-\pi_{T}^{*}\right)$, plotted as a function of the cost of enhanced design, $c_{D}$.

impact of the behavioral effect of enhanced design. Whether quick response and enhanced design are complements or substitutes hinges on whether this is beneficial to the firm. If the behavioral effect of enhanced design results in a decrease in firm profit (which happens if $c_{D}$ is small), then the moderating presence of quick response's behavioral effect leads to complementarity. If, on the other hand, the behavioral effect of enhanced design leads to an increase in firm profit (which happens if $c_{D}$ is large), then quick response's behavioral effect reduces the incremental impact of enhanced design along the behavioral dimension. If this substitution effect is sufficiently strong, it can overwhelm the complementary interaction along the operational dimension and lead to a net substitution effect.

This discussion may lead one to conclude that quick response and enhanced design are more likely to be substitutes as consumers become more strategic (i.e., as $\delta$ grows large). This need not be the case, however. The reason is that it is not obvious how a more strategic customer population impacts the complementarity of the practices along the operational dimension. Recall that while this interaction exists even if $\delta=0$, it is not independent of $\delta$. The complicated way that quick response and enhanced design interact with one another, and the question of how $\delta$ impact the net interaction, motivates the numerical study in the following section. 


\section{Numerical Study}

The preceding analysis leads to several interesting questions. First: when the conditions Theorem 1 are violated (specifically the $c_{D}=0$ condition), how pervasive is the complementarity result? Second: what is the magnitude of the complementarity effect? Third: how is the complementarity effect impacted by changes in the various parameter values (in particular, $\delta$, the consumer discount factor)? And fourth: under what conditions are the various production systems most valuable? Because the equilibrium expressions for prices, inventory levels, and profits are complex and difficult to decipher analytically, we employ an extensive numerical study in $\S \S 8.1-8.2$ to answer these questions. Lastly, $§ 8.3$ presents a numerical analysis of an extension to our base mode: designdependent clearance prices.

\subsection{The Value of Fast Fashion}

The study consists of 8,019 total instances resulting from every possible combination of the values listed in Table 3. These parameters represent a wide range of plausible values, chosen to represent realistic scenarios from the fashion apparel industry. The coefficient of variation of demand $(\sigma / \mu)$ equals 0.5, 0.75, or 1 (Hammond and Raman 1994 report similar values, e.g., less than one, in the context of skiwear). Maximum gross margins (i.e., $(v-c) / v$ in the standard design systems and $\left(v+m-c-c_{D}\right) /(v+m)$ in the enhanced design systems) range from $33 \%$ to $83 \%$ (actual gross margins depend on the equilibrium selling price and can even be negative in "unprofitable" enhanced design systems). These figures are in-line with the reported gross margins from the annual filings of many fashion apparel firms. ${ }^{11}$ Enhanced design and quick response each incur $10 \%$ to $50 \%$ cost premiums (thus, fast fashion incurs $20 \%$ to $100 \%$ cost premiums), and "hot" products generated with enhanced design generate between $10 \%$ and $30 \%$ more consumer value than safe products created without enhanced design. While these parameters are naturally more difficult to match to industry data, we believe they are plausible given the costs of local production versus outsourced production and transportation (e.g., a fast fashion designed product can be anywhere from $20 \%$ to $100 \%$ more expensive than a traditional product).

Note that the entire sample consists of instances with $c_{D}>0$, meaning the entire numerical

\footnotetext{
${ }^{11}$ A search on Google Finance for fashion retailer gross margins in annual reports shows ranges in the interval $38 \%$, for Nordstrom, to 70-80\%, for leather-goods makers like Coach and Piquadro.
} 


\begin{tabular}{c|c}
\hline \hline Parameter & Values \\
\hline Demand Distribution & Normal \\
$\mu$ & 150 \\
$\sigma$ & $\{75,112.5,150\}$ \\
$v$ & 8 \\
$m$ & $\{1,2,3\}$ \\
$c$ & $\{2,3,4\}$ \\
$c_{D}$ & $\{0.1 c, 0.3 c, 0.5 c\}$ \\
$c_{Q}$ & $\{0.1 c, 0.3 c, 0.5 c\}$ \\
$s$ & $\{0.75 c, 0.85 c, 0.95 c\}$ \\
$\delta$ & $\{0,0.1,0.2, \ldots, 1\}$ \\
\hline \hline
\end{tabular}

Table 3. Parameter values used in numerical experiments.

analysis lies outside the scope of Theorem 1. For each parameter combination, we calculated the equilibrium under all four systems and determined expected prices and profits. Even though the sufficient conditions for complementarity from Theorem 1 were not satisfied by any parameter combination, the complementarity result held virtually always: in 7,969 cases (99.4\% of the sample), we observed that the value of a fast fashion system (the increase in profit over the traditional system) was weakly greater than the combined value of quick response and enhanced design operating alone. $^{12}$ Furthermore, the fast fashion system was optimal (provided the greatest expected profit) in 6,985 cases $(87.1 \%)$.

We first consider the magnitude of the complementarity effect. In particular, we analyze the complementarity effect as a function of the consumer discount factor $(\delta)$. Figure 5 plots the percentage increase in firm profit (relative to the base case of the traditional system) from each of the three alternative strategies, averaged over our entire sample. Included in the graph is a plot of the sum of the values of quick response and enhanced design, expressed as a percentage increase in firm profit; if the value of fast fashion lies above this sum, then the complementarity effect exists. As the figure demonstrates, when $\delta$ is small (consumers are not very strategic), the complementarity effect is also small; when $\delta$ is large (consumers are very strategic) the complementarity effect is dramatic, with fast fashion adding about twice as much profit as the combined values of enhanced design and production. Hence, the magnitude of complementarity appears to increase as consumers

\footnotetext{
${ }^{12}$ In the 50 cases in which enhanced design and rapid production were not complementary, we observed that enhanced design provided strongly negative value on its own (because the cost of enhanced design was too high to justify the relatively small price increase), as one might expect from the discussion in $\S 7$. However, 2,898 cases yielded negative value for enhanced design, but nevertheless the complementarity result held in 2,848 of these.
} 


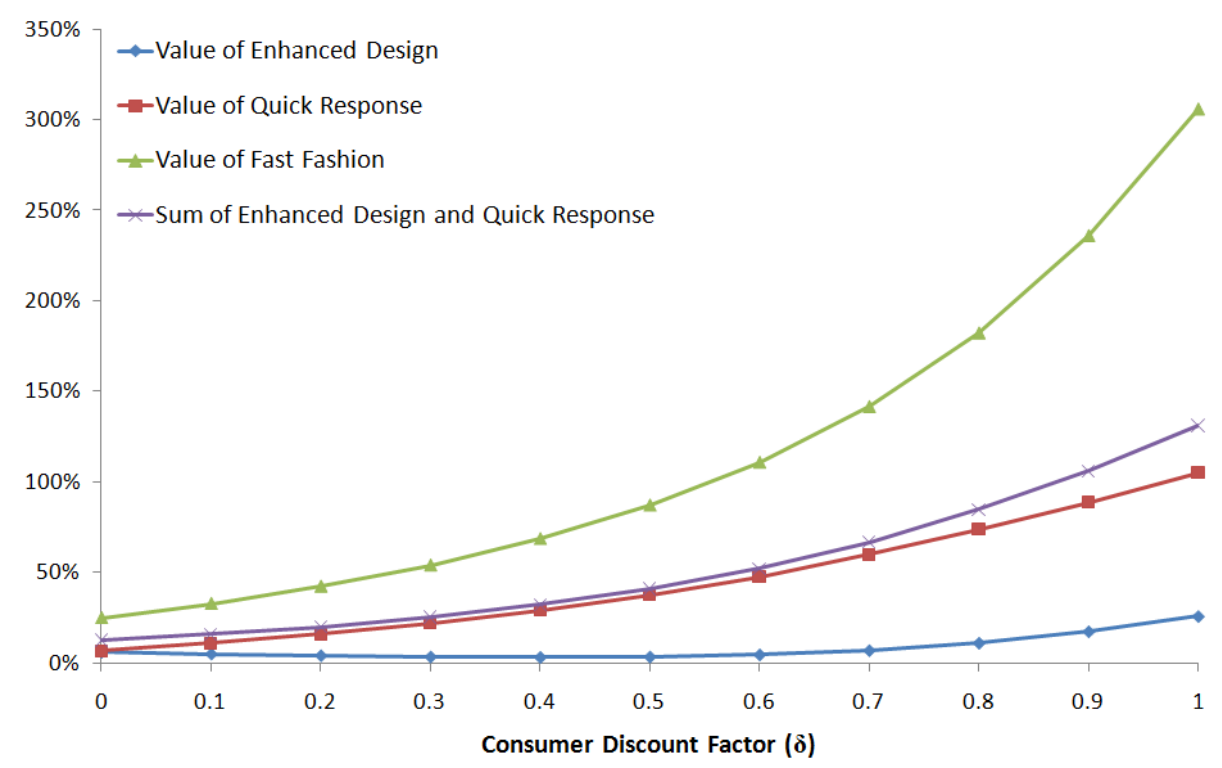

Figure 5. The percentage increase in expected profit (over the traditional system) in each system as a function of the consumer discount factor $(\delta)$, averaged over the entire sample.

become more strategic, implying that fast fashion systems are most valuable when consumers are very strategic. ${ }^{13}$

We stress, however, that it isn't always the case that complementarity increases as $\delta$ increases: the ratio between the value of fast fashion and the sum of the values of enhanced design and quick response as a function of $\delta$ is not monotonic. On average, though, if $\delta$ is zero (consumers are myopic), then fast fashion is roughly 1.98 times as valuable as the sum of the independent systems; when consumers are perfectly strategic $(\delta=1)$, it is more than 2.3 times as valuable as the sum of the independent strategies. Thus, despite the fact that substitution is only possible via interaction along the behavioral dimension, generally speaking the complementarity is strongest if customers are very strategic. This is a consequence of the fact the behavioral effects of quick response and enhanced design are complementary if $c_{D}$ is sufficiently small (and, in our sample, this occurs in most instances), and this complementarity effect strengthens as behavioral factors become more important to the system (i.e., as $\delta$ grows large). We conclude from this observation that quick response and enhanced design experience the strongest complementarity when $\delta$ is large (customers are very strategic) and the variable costs of enhanced design are not too large.

\footnotetext{
${ }^{13}$ Indeed, it is possible to find parameter combinations for which expected profit is zero in the traditional production system, and positive in one or more of the alternative systems. Thus, it is possible for the systems to possess "infinite value," in relative terms, though we designed the numerical study to avoid these cases to facilitate analysis of the percentage profit increase resulting from the three alternative strategies.
} 

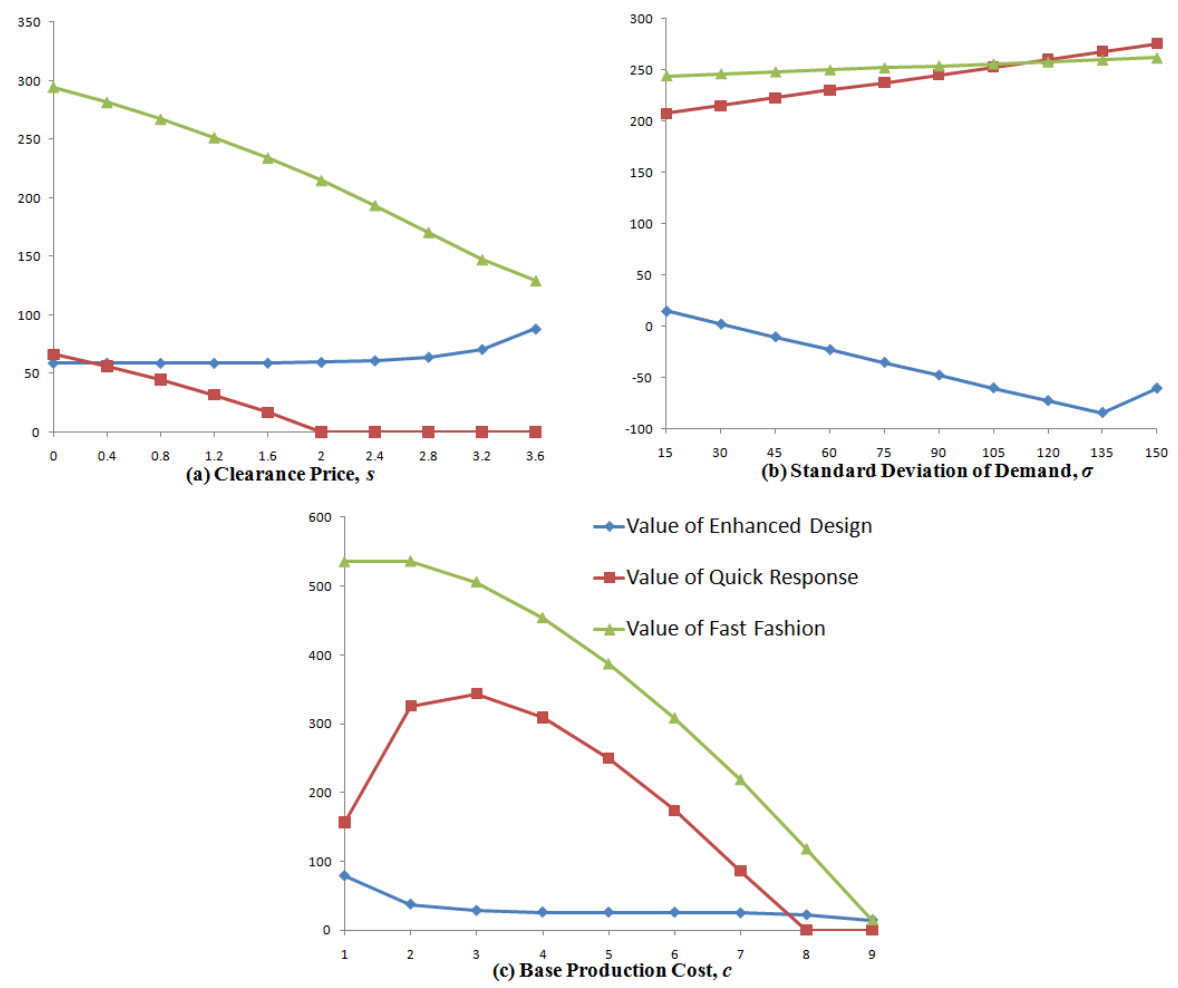

Figure 6. Examples of the value of the alternative product strategies (incremental increase in profit over the traditional system) as a function of three parameters.

\subsection{When is Fast Fashion Most Valuable?}

The preceding discussion focused on when the complementarity effect holds, but understanding the conditions that dictate optimality of the various production systems can help guide managers on when to invest in fast fashion capabilities. Thus, in this section, we discuss the following more specific question: when is a fast fashion system most valuable? To address this question, we have chosen to examine a few representative numerical examples, rather than using a broad, large-scale study as in the previous subsection. The results of these examples are depicted graphically in Figure 6. Each subfigure plots the value of an alternative production strategy (i.e., in the incremental increase in profit over the traditional system) as a function of a different problem parameter.

Figure 6 a plots the value of the three strategies as a function of $s$, the clearance price. In the example, demand is normally distributed with $\mu=150$ and $\sigma=75$, and the remaining parameters are: $v=10, m=3, c=4, c_{D}=1, c_{Q}=2$, and $\delta=1$. Observe first that the values of quick response (squares) and fast fashion (triangles) are both decreasing in $s$; this is natural, since it becomes less important to reduce overage costs by adopting rapid production capabilities as the 
clearance or salvage cost increases. Interestingly, the value of enhanced design (diamonds) is increasing in $s$. This is because of behavioral effects: the greater the clearance price, the less incentive consumers have to wait for the sale, and hence the firm can extract a greater fraction of the value increase $(m)$ when adopting enhanced design. Thus, for small $s$, quick response may be preferred to enhanced design, but for large $s$ this relationship can be reversed. ${ }^{14}$ We note that given our model assumptions, fast fashion always yields a profit at least as great as the profit in an enhanced design system, so it is impossible for enhanced design to be preferred to fast fashion; nevertheless, the gap between the two decreases as the clearance price increases. Consequently, fast fashion is of greatest incremental value when clearance prices are expected to be low.

Figure $6 \mathrm{~b}$ plots the value of each strategy as a function of the standard deviation of demand, $\sigma$. In the example, demand is normally distributed with $\mu=150$, and the remaining parameters are: $v=10, m=2, c=4, c_{D}=2.6, c_{Q}=1, s=2$ and $\delta=1$. As one would expect, the two strategies employing rapid production capabilities are most valuable when demand is highly variable. The value of enhanced design, however, is decreasing ${ }^{15}$ in $\sigma$. This is because enhanced design is accompanied by potentially greater production costs; combining these greater production costs with greater demand variability leads to greater inventory overage costs, and hence a decreasing value effect. Note that this effect is also present for fast fashion; as a result, the value of fast fashion increases slower as a function of $\sigma$ than the value of quick response, and it can be the case that fast fashion is optimal for low $\sigma$ while quick response is optimal for very high $\sigma$. Enhanced design must be very costly for this to be a significant effect, however-in the example, $c_{D}=2.6>2=m$.

Lastly, Figure 6c plots the value of each strategy as a function of the base production cost, c. In the example, demand is normally distributed with $\mu=150$ and $\sigma=75$, and the remaining parameters are: $v=10, m=2, c_{D}=1, c_{Q}=1, s=0.5$ and $\delta=1$. First observe that the value of quick response is concave in $c$-at first increasing, then decreasing. The value is initially increasing because greater $c$ implies greater inventory overage costs, meaning the firm can benefit by moving to a quick response system which reduces overage. Moreover, quick response always reduces the chance of a clearance sale, which gives a behavioral benefit as well. However, both of these factors

\footnotetext{
${ }^{14}$ Of course, it is possible to construct examples in which the value of quick response always dominates the value of enhanced design, and vice versa. The figure depicts just one possible ordering.

${ }^{15}$ Although there is an increasing portion for high $\sigma$, this is in fact a region where enhanced design is so costly that the optimal production quantity is zero. Because profit in the traditional system continues to decrease as a function of $\sigma$, the incremental value $\pi_{D}^{*}-\pi_{T}^{*}=-\pi_{T}^{*}$ begins to increase.
} 
are diminished at high $c$ : in this case, the margins are so low that the additional cost of a unit procured using quick response $\left(c+c_{Q}\right)$ essentially eliminates firm profit, leading to a preference for traditional production. Furthermore, when costs are high the chance of a clearance sale is already low in the traditional system, meaning there is limited behavioral benefit from quick response at high $c$.

The value of enhanced design, on the other hand, is decreasing in $c$. This, again, is due to the fact that the behavioral advantages of enhanced design are lower when the production cost is higher and hence the chance of a clearance sale is already low; moreover, if costs are already high, the firm may be unwilling to tolerate additional costs resulting from enhanced design. The behavior of fast fashion as a function of $c$ is, as one would expect, somewhere between the behaviors of quick response and enhanced design. We observe that this implies fast fashion is most valuable for low to moderate production cost items. ${ }^{16}$ This may at first appear counter-intuitive: fast fashion production, which minimizes waste and maximizes consumer value, would seemingly appear most valuable when costs are high and hence waste is expensive and margins low. Hence, the behavioral consequences of fast fashion that we identify lead to new, surprising effects that run counter to conventional operational wisdom.

To summarize the results of this section, we have noted that fast fashion is most valuable for low cost items with high demand variability and low clearance prices-precisely the scenario observed in the fashion apparel industry.

\subsection{Design-Dependent Clearance Price}

In the preceding analytical and numerical results, we assumed that the clearance or salvage price $(s)$ was independent of the production system used by the firm. In particular, the enhanced design and fast fashion systems had no greater clearance price than the quick response and traditional systems, despite the purported enhancements to product design resulting in greater consumer value in the former two production modes. One might reasonably argue, though, that enhanced design results in changes to the product that yield an increase in the clearance price proportional to the increase in consumer value, $m$. In this section we consider this possibility, modeling the clearance price in the enhanced design and fast fashion systems as equal to $s+\gamma m$, where $\gamma \in[0,1]$ represents

\footnotetext{
${ }^{16}$ The value of fast fashion need not be decreasing in $c$; like quick response, the value can be more concave in $c$.
} 
the residual fraction of enhanced design's incremental value that carries into the clearance market.

This change complicates the analytical comparisons of the various systems significantly. The reason for this is that a higher clearance price, all else being equal, increases the firm's optimal inventory level, thereby increasing the probability of a clearance sale and hence increasing consumer incentives to delay purchasing-this means the firm must reduce prices to induce consumers to buy early. At the same time, consumers must pay a higher clearance price and so consumer utility (conditional on obtaining a unit) is reduced-this, in contrast to the preceding effect, means the firm can raise prices and still induce early purchasing. Which effect dominates is unclear, and consequently, the total effect of higher clearance prices on equilibrium full prices and inventory is not obvious. Moreover, even if higher clearance prices have an unambiguous effect on the equilibrium full price, the ultimate impact on profit is not clear; if, e.g., the increased availability effect dominates and full prices are decreasing in $\gamma$, the firm's salvage value is increasing in $\gamma$, meaning full price revenues are decreasing and clearance revenues are increasing in $\gamma$, with the net effect unclear. Hence, in this section, we resort to numerical analysis to study this issue.

We first discuss selected examples similar to our approach $\S 8.2$. In these examples, demand is normal with $\mu=150$ and $\sigma=75$. In addition, $v=10, m=1, c=3, c_{D}=0.3, c_{Q}=1.5$, and $\delta=1$. Figure 7a illustrates the incremental value of each of the alternative production systems (i.e., the increase in profit over the traditional system) as a function of $\gamma$, the residual value parameter, when $s=0.3$. Note that the value of quick response is independent of $\gamma$, since this system does not possess enhanced design features. Our first observation is that the value of enhanced design is not monotonic in $\gamma$ (though the variation is slight); rather, it is roughly concave, peaking around $\gamma=0.5$. Thus, the two counteracting forces we described above (an increase in $\gamma$ leading to a simultaneous decrease in the selling price and increase in salvage values) dominate at different times: for small $\gamma$, the increase in clearance revenues dominates and leads to greater overall profit, whereas for large $\gamma$, the decrease in the equilibrium prices dominates and leads to lower profits.

Next, observe that the value of fast fashion is decreasing ${ }^{17}$ in $\gamma$, and at a faster rate than the value of enhanced design. This latter feature is characteristic of the examples we have examined. The greater impact of $\gamma$ on the value of fast fashion appears to be rooted in the fact that the

\footnotetext{
${ }^{17}$ We note that the value of fast fashion need not be strictly decreasing in $\gamma$; as with enhanced design, a more concave shape is possible.
} 

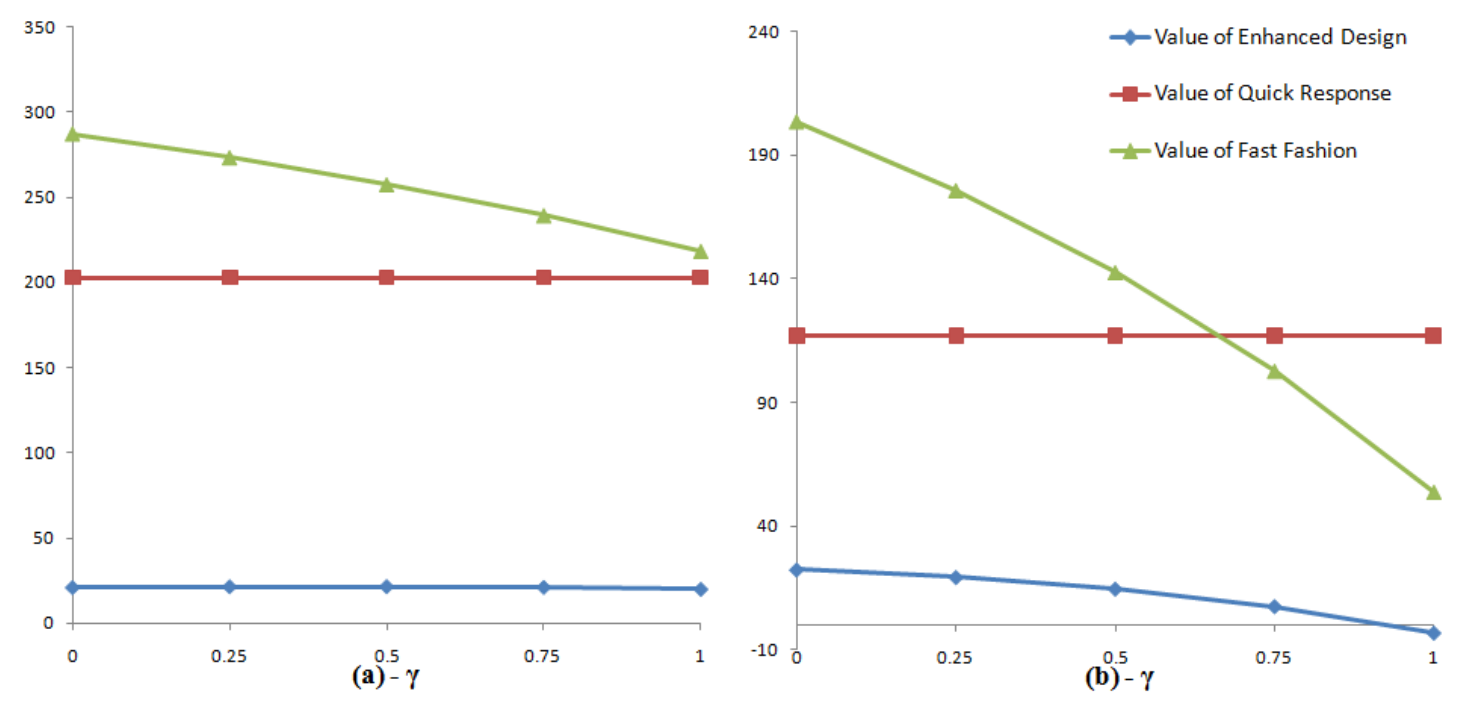

Figure 7. An example of the incremental value of the various production systems as a function of $\gamma$ when $s=0.3(\mathbf{a})$ and when $s=1.5(\mathbf{b})$.

equilibrium in-stock probability is independent of the full price in the fast fashion system; hence, increasing $\gamma$ unambiguously increases this term. Greater in-stock probabilities in turn lead to lower equilibrium prices and profits. On the other hand, in the enhanced design system, the in-stock probability depends on the price as well as the salvage value; hence, greater salvage values leading to greater consumer incentive to delay may lead to lower prices, which in turn moderates the impact on the in-stock probability. The consequence of this result is that the value of fast fashion appears to be most sensitive to the assumptions on clearance price analyzed in this section; enhanced design has (relatively) minimal variation as a function of $\gamma$.

In Figure 7a, fast fashion is always optimal, and the complementarity effect holds except at very high $\gamma$-in other words, unless a significant portion of the value increase $m$ carries into the salvage period, complementarity holds and fast fashion is optimal. For very high $\gamma$, complementarity no longer holds because of the severe effect of high clearance prices on the value of fast fashion. This is representative of the numerical examples we have explored, although we note that the "threshold $\gamma$ " above which complementarity ceases to hold can vary substantially; in Figure 7a, it is approximately 0.95 , while in Figure $7 \mathrm{~b}$, which is an identical example save for $s=1.5$, the threshold $\gamma$ is approximately 0.5 .

To test this logic on a larger scale, we extended the full scale numerical study using the parameter combinations in Table 3 to allow for $\gamma>0$. To ensure finite solutions in all possible combinations, 
we require $\gamma<0.1$ (i.e., $\gamma>0.1$ may result in negative overage costs in some instances). Using $\gamma \in\{0.05,0.07,0.09\}$, we found that complementarity held in 23,792 out of 24,057 cases, or $98.89 \%$ of the sample, compared to $99.4 \%$ of the sample with $\mathbf{\square}=0$. We conclude that our primary result, that quick response and enhanced design are complements, continues to hold with design-dependent salvage values if $\gamma$ is not too large.

\section{Conclusion}

With the success of fast fashion retailers, an increasing amount of attention-both academic and practical-has been paid to these innovative firms. In this paper, we present a modeling framework that allows us to capture and isolate the key aspects that define a fast fashion system: enhanced design efforts and quick response capabilities. By employing this approach, we analyze four potential operating systems: traditional systems (with standard design efforts and slow production), quick response systems, enhanced design systems, and fast fashion systems (with both enhanced design and quick response), and characterize equilibrium inventory levels, prices, and consumer purchasing behavior in each case.

We focus much of our discussion on the issue of whether quick response and enhanced design are complements or substitutes. We find that, while it is possible for the two practices to be substitutes, it is much more likely that they are complements. The reason is that there are multiple forces impacting sales and prices which determine complementarity. In the vast majority of our numerical cases (over 99\%), the complementarity factors (significantly) outweigh the substitution factors, leading enhanced design and quick response to be overall complements.

This result occurs despite the fact that, as we alluded to earlier, we have ignored a crucial aspect of how enhanced product design interacts with quick response: namely, that enhanced design may simply be more effective if production leadtimes are shorter. If, for example, the production leadtime is six months, then no matter how much effort the firm places on product design, it still must finalize design well in advance of the selling season, meaning it may miss important trends and changes in consumer preferences. On the other hand, if the production leadtime is one month, then design may be finalized much later, allowing the firm to pursue changing trends in a much more agile and responsive manner. Consequently, the potential value of enhanced design-all else 
being equal-can be greater if the firm has achieved quick response.

We find that, even controlling for the latter complementarity effect (assuming that it is zero), the two practices are almost always complements. Thus, the complementarity of these two strategies does not, in general, depend on the fact that production leadtime reduction allows a firm to delay its design decisions. However, if we were to include this effect in concert with the other forces we have described, the complementarity of enhanced design and quick response would be even more dramatic, a fact which leads us to conclude that there is substantial value-operationally and behaviorally-from adopting a fast fashion approach.

The fact that enhanced design and quick response are complements-and that the magnitude of complementarity increases as customers become more strategic-helps to explain how even seemingly costly systems can be profitable. European fast fashion retailers such as Zara, H\&M, and Benetton, for example, employ large staffs of in-house designers and even use costly local labor and expedited shipping methods when necessary. While this seemingly puts these firms at a heavy cost disadvantage, they manage to reap additional benefits by minimizing strategic behavior, more so even than employing either production strategy by itself.

Naturally, when choosing whether to implement one of the strategies we describe, a firm must evaluate fixed costs in addition to the variable costs and operating profits that we analyze. However, the fact remains that even when fixed costs are accounted for, the value of the fast fashion system, relative to the alternative systems, generally increases as consumers become more patient (and hence more strategic in their purchasing behavior), a fact which justifies the use of sophisticated production systems capable of enhanced design and quick response in markets characterized by savvy consumer populations. Crucially, the magnitude of complementarity between the base strategies of fast fashion systems is greatest if customers are very strategic and the marginal production cost impact of enhanced design is small, meaning we would expect to see most fast fashion implementations in precisely these conditions. This prediction may be empirically testable, which could present interesting opportunities for future research.

In addition, there are a number of other (non-operational) reasons why a firm might adopt a fast fashion strategy, including: competitive and marketing issues (e.g., fast fashion as a competitive distinction), market positioning (to high-end or fashion-conscious consumers), and political or social concerns (e.g., localized production as an act of social responsibility or public relations by the firm). 
All of these reasons, and doubtless many more, influence the value of fast fashion. However, as our model shows, an important consequence of fast fashion product is its impact on consumer purchasing behavior and the operational efficiency of the firm. While quick response and enhanced design practices are not suited to every industry or every product, in cases where the strategies are feasible and not prohibitively expensive, the reward for implementing such systems simultaneously can be significant, particularly when consumers are sophisticated.

Acknowledgements: The authors thank Serguei Netessine, Senthil Veeraraghavan, and conference participants at the INFORMS Annual Meeting in Washington, D.C. and the 2010 London Business School Innovation in Operations Conference for helpful feedback, as well as the department editor, associate editor, and three anonymous referees for numerous comments which greatly improved the paper.

\section{A Appendix: Proofs}

We introduce the following notation, which we use throughout the analysis: let $(x)^{+}=\max (x, 0)$, let $L(q)=\mathbb{E}(N-q)^{+}$be the expected lost sales (excess demand above $q$ ) and let $I(q)=\mathbb{E}(q-N)^{+}$ be the expected leftover inventory (excess inventory above $N$ that is cleared at the sale price $s$ ).

Proof of Lemma 1. As discussed in the discussion preceding the lemma, the only viable equilibrium is one in which all consumers attempt to purchase at the full price. Hence, for this proof (and all remaining proofs) we restrict our attention to that case. The profit function in (??) is the familiar newsvendor formula yielding an optimal inventory level (given a particular price $p$ ) satisfying $F(q)=(p-c) /(p-s)$. Thus, this equilibrium is viable if consumers have incentive to purchase early, i.e., if

$$
v-p \geq \delta \phi(v-s),
$$

if the firm chooses the optimal inventory level, $F\left(q_{T}^{*}\right)=(p-c) /(p-s)$, and if expectations of consumers are rational. Rationality of consumer expectations implies that $\phi$ is the actual probability that a consumer who unilaterally deviates from the equilibrium (by attempting to buy during the clearance sale) obtains the product-in other words, the probability that a single consumer who buys late gets the product conditional on all other consumers (from the market represented by $N$ ) buying early. This occurs if and only if the firm has sufficient inventory, $q_{T}^{*}$, to cover the entire market, $N$. Hence, $\phi=\operatorname{Pr}\left(N \leq q_{T}^{*}\right)=F\left(q_{T}^{*}\right)$. When choosing the price $p$, the firm maximizes its profit by selecting the highest price that satisfies (2), which implies the optimal pricing policy is to set a full price equal to $p_{T}^{*}=v-\delta \phi(v-s)$. Combining this expression with the $\phi=F\left(q_{T}^{*}\right)$ requirement yields

$$
p_{T}^{*}=v-\delta \frac{p_{T}^{*}-c}{p_{T}^{*}-s}(v-s)
$$

Simplifying this expression yields

$$
p_{T}^{*}=\frac{A(v) \pm \sqrt{A(v)^{2}-4 B(v, c)}}{2} .
$$


The lower candidate equilibrium sale price results in $p_{T}^{*}<c$, and hence is unsupportable; thus, a unique equilibrium exists which satisfies the conditions in the lemma.

Proof of Lemma 2. By the same logic from Lemma 1, the only possible candidate equilibrium is one in which all consumers attempt to purchase at the full price. Suppose $p \geq c+c_{Q}$. In such an equilibrium, the firm's expected profit with quick response as a function of the initial inventory procurement $(q)$ and price $p$ is

$$
\pi_{Q}(q, p)=(p-c) \mu-c_{Q} L(q)-(c-s) I(q) .
$$

This function is concave in $q$, and the unique optimal inventory level is given by

$$
q_{Q}^{*}=F^{-1}\left(\frac{c_{Q}}{c+c_{Q}-s}\right) \text {. }
$$

Note that this quantity is independent of the selling price. Recall that consumers purchase early if

$$
v-p \geq \delta \phi(v-s)
$$

If the firm behaves optimally and if consumer expectations are rational, then $\phi=F\left(q_{Q}^{*}\right)=$ $c_{Q} /\left(c+c_{Q}-s\right)$. Hence, the maximum price that induces consumers to purchase prior to the sale by making (3) hold with equality is given by (1). Alternatively, if $p<c+c_{Q}$, the firm will never use the option to procure additional inventory, meaning the profit function and equilibrium analysis reduce to that analyzed in Lemma 1.

Proof of Lemma 3. By observing the expressions for the equilibrium prices, note that the price is higher in the $Q$ system than the $T$ system if and only if the probability that a customer obtains a unit at the sale price $(\phi)$ is lower in the $Q$ system. This happens if

$$
\frac{c_{Q}}{c+c_{Q}-s}<\frac{p_{T}^{*}-c}{p_{T}^{*}-s} .
$$

Rearranging the terms, this reduces to $c_{Q}<p_{T}^{*}-c$.

Proof of Lemma 7. Comparing the equilibrium prices from Lemmas 2 and 6 , it is easy to see that $p_{F}^{*}>p_{Q}^{*}$. Comparing prices from Lemmas 4 and 6 , observe that the price in the $F$ system is greater than the price in the $D$ system if and only if the equilibrium $\phi$ is lower in the $F$ system, i.e., if

$$
\frac{c_{Q}}{c+c_{D}+c_{Q}-s}<\frac{p_{D}^{*}-c-c_{D}}{p_{D}^{*}-s} .
$$

Rearranging the terms, the inequality holds if

$$
\left(p_{D}^{*}-c-c_{D}\right)\left(c+c_{Q}+c_{D}-s\right)-c_{Q}\left(p_{D}^{*}-s\right)>0,
$$

which, in turn, reduces to the condition

$$
\left(c+c_{D}-s\right)\left(p_{D}^{*}-c-c_{Q}-c_{D}\right)>0 .
$$

Since $c+c_{D}-s>0$, a necessary and sufficient condition for the relationship to hold is $p_{D}^{*}-c-$ $c_{Q}-c_{D}>0$. The result that $p_{F}^{*}>p_{T}^{*}$ then follows from Lemma 5 if $p_{D}^{*}>c+c_{D}+c_{Q}$ holds.

Proof of Theorem 1. Quick response and enhanced design are complements if $\pi_{F}^{*}-\pi_{Q}^{*} \geq$ $\pi_{D}^{*}-\pi_{T}^{*}$. Let $S(x)=\mu-L(x)$ be the expected sales given an inventory level of $x$. Note that 
$S(x) \leq \mu$ for any $x$. When $c_{D}=0$, the equilibrium profit in each of the four systems is

$$
\begin{aligned}
\pi_{T}^{*} & =\left(p_{T}^{*}-c\right) S\left(q_{T}^{*}\right)-(c-s) I\left(q_{T}^{*}\right), \\
\pi_{Q}^{*} & =\left(p_{Q}^{*}-c\right) \mu-\left(c_{Q}\right) L\left(q_{Q}^{*}\right)-(c-s) I\left(q_{Q}^{*}\right), \\
\pi_{D}^{*} & =\left(p_{D}^{*}-c\right) S\left(q_{D}^{*}\right)-(c-s) I\left(q_{D}^{*}\right), \\
\pi_{F}^{*} & =\left(p_{F}^{*}-c\right) \mu-\left(c_{Q}\right) L\left(q_{F}^{*}\right)-(c-s) I\left(q_{F}^{*}\right) .
\end{aligned}
$$

Let $\pi_{\Omega}(q)$ be the profit in system $\Omega \in\{T, D, P, F\}$ at quantity level $q$. Observe that $\pi_{F}^{*}-\pi_{Q}^{*}=$ $\pi_{F}\left(q_{F}^{*}\right)-\pi_{Q}\left(q_{Q}^{*}\right) \geq \pi_{F}\left(q_{Q}^{*}\right)-\pi_{Q}\left(q_{Q}^{*}\right)$, and hence

$$
\begin{aligned}
\pi_{F}^{*}-\pi_{Q}^{*} & \geq\left(p_{F}^{*}-p_{Q}^{*}\right) \mu+c_{Q}\left(L\left(q_{Q}^{*}\right)-L\left(q_{Q}^{*}\right)\right)+(c-s) I\left(q_{Q}^{*}\right)-(c-s) I\left(q_{Q}^{*}\right) \\
& =\left(p_{F}^{*}-p_{Q}^{*}\right) \mu .
\end{aligned}
$$

Similarly, $\pi_{D}^{*}-\pi_{T}^{*}=\pi_{D}\left(q_{D}^{*}\right)-\pi_{T}\left(q_{T}^{*}\right) \leq \pi_{D}\left(q_{D}^{*}\right)-\pi_{T}\left(q_{D}^{*}\right)$, which implies

$$
\begin{aligned}
\pi_{D}^{*}-\pi_{T}^{*} & \leq\left(p_{D}^{*}-p_{T}^{*}\right) S\left(q_{D}^{*}\right)-(c-s) I\left(q_{D}^{*}\right)+(c-s) I\left(q_{D}^{*}\right) \\
& =\left(p_{D}^{*}-p_{T}^{*}\right) S\left(q_{D}^{*}\right) .
\end{aligned}
$$

Because $S(x) \leq \mu$ for any $x$, it follows that

$$
\left(p_{D}^{*}-p_{T}^{*}\right) \mu \geq\left(p_{D}^{*}-p_{T}^{*}\right) S\left(q_{D}^{*}\right) \geq \pi_{D}^{*}-\pi_{T}^{*} .
$$

It follows that complementarity holds if $p_{F}^{*}-p_{Q}^{*} \geq p_{D}^{*}-p_{T}^{*}$, or equivalently if $p_{F}^{*}-p_{D}^{*} \geq p_{Q}^{*}-p_{T}^{*}$. Observe that when $m=0, p_{F}^{*}-p_{D}^{*}=p_{Q}^{*}-p_{T}^{*}$. (That is, if both $c_{D}=0$ and $m=0$, fast fashion is equivalent to quick response and enhanced design is equivalent to the traditional system.) Because $p_{Q}^{*}$ and $p_{T}^{*}$ are independent of $m$, to show $p_{F}^{*}-p_{D}^{*} \geq p_{Q}^{*}-p_{T}^{*}$ for all $m>0$ it is sufficient to show $p_{F}^{*}-p_{D}^{*}$ is increasing in $m$. Substituting $c_{D}=0$ into the equilibrium price equations from Lemmas 4 and 6 , we have

$$
\begin{aligned}
p_{D}^{*}= & \frac{(v+m)(1-\delta)+(1+\delta) s}{2} \\
& +\frac{\sqrt{((v+m)(1-\delta)+(1+\delta) s)^{2}-4(s(v+m)-\delta c(v+m-s))}}{2}, \\
p_{F}^{*}= & v+m-\delta \frac{c_{Q}}{c+c_{Q}-s}(v+m-s) .
\end{aligned}
$$

The difference between these expressions is

$$
p_{F}^{*}-p_{D}^{*}=(v+m-s)\left(1-\delta \frac{c_{Q}}{c+c_{Q}-s}-\frac{(1-\delta)}{2}-\frac{1}{2} \sqrt{1+\delta^{2}-2 \delta \frac{v+m+s-2 c}{v+m-s}}\right) .
$$

Differentiating with respect to $m$,

$$
\begin{aligned}
\frac{d}{d m}\left(p_{F}^{*}-p_{D}^{*}\right)= & \left(1-\delta \frac{c_{Q}}{c+c_{Q}-s}-\frac{(1-\delta)}{2}-\frac{1}{2} \sqrt{1+\delta^{2}-2 \delta \frac{v+m+s-2 c}{v+m-s}}\right) \\
& +\frac{\delta(c-s)}{(v+m-s) \sqrt{1+\delta^{2}-2 \delta \frac{v+m+s-2 c}{v+m-s}}} .
\end{aligned}
$$


The second term is clearly non-negative; the first term is non-negative if and only if $p_{F}^{*} \geq p_{D}^{*}$. From Lemma 5, this occurs if $c_{Q}<p_{D}^{*}-c$. Observing that $p_{D}^{*}$ is decreasing in $\delta$, and substituting $\delta=1$ into the expression for $p_{D}^{*}$, we see that a sufficient condition for $c_{Q}<p_{D}^{*}-c$ is $c_{Q}<$ $s+\sqrt{(v+m-s)(c-s)}-c$. This implies that if $c_{D}=0$ and $c_{Q}$ is sufficiently small, $p_{F}^{*}-p_{D}^{*}$ is increasing in $m$. The complementarity result $\left(\pi_{F}^{*}-\pi_{Q}^{*} \geq \pi_{D}^{*}-\pi_{T}^{*}\right)$ follows.

\section{References}

Anand, K., K. Girotra. 2007. The strategic perils of delayed differentiation. Management Sci. 53(5) $697-712$.

Aviv, Y., A. Pazgal. 2008. Optimal pricing of seasonal products in the presence of forward-looking consumers. Manufacturing Service Oper. Management 10(3) 339-359.

Bulow, J. I. 1982. Durable-goods monopolists. The Journal of Political Economy 90(2) 314-332.

Cachon, G. P., R. Swinney. 2009. Purchasing, pricing, and quick response in the presence of strategic consumers. Management Sci. 55(3) 497-511.

Caro, F., J. Gallien. 2009. Inventory management of a fast-fashion retail network. Oper. Res. Forthcoming.

Caro, F., V. Martínez-de-Albéniz. 2009. The impact of quick response in inventory-based competition. Manufacturing Service Oper. Management Forthcoming.

Coase, R. H. 1972. Durability and monopoly. J. Law \& Econ. 15(1) 143-149.

Eppen, G. D., A. V. Iyer. 1997. Improved fashion buying with Bayesian updating. Oper. Res. 45(6) 805-819.

Feitzinger, E., H. Lee. 1997. Mass customization at Hewlett-Packard: The power of postponement. Harvard Business Review 75(1) 116-121.

Ferdows, K., M. A. Lewis, J. A. D. Machuca. 2004. Rapid-fire fulfillment. Harvard Business Review 82(11) 104-110.

Fisher, M., K. Rajaram, A. Raman. 2001. Optimizing inventory replenishment of retail fashion products. Manufacturing Service Oper. Management 3(3) 230-241.

Fisher, M., A. Raman. 1996. Reducing the cost of demand uncertainty through accurate response to early sales. Oper. Res. 44(1) 87-99.

Ghemawat, P., J. L. Nueno. 2003. ZARA: Fast Fashion. Case Study, Harvard Business School.

Goyal, M., S. Netessine. 2007. Strategic technology choice and capacity investment under demand uncertainty. Management Sci. 53(2) 192-207.

Hammond, J. H., A. Raman. 1994. Sport Obermeyer Ltd. Case Study, Harvard Business School.

Iyer, A. V., M. E. Bergen. 1997. Quick response in manufacturer-retailer channels. Management Sci. 43(4) 559-570.

Lee, H., C. Tang. 1997. Modelling the costs and benefits of delayed product differentiation. Management Sci. 43(1) 40-53. 
Li, Q., A. Ha. 2008. Reactive capacity and inventory competition under demand substitution. IIE Transactions 40(8) 707-717.

Liu, Q., G. van Ryzin. 2008. Strategic capacity rationing to induce early purchases. Management Sci. 54(6) 1115-1131.

Meichtry, S. 2007. Benetton picks up the fashion pace. The Wall Street Journal.

Milgrom, P., J. Roberts. 1990. The economics of modern manufacturing: Technology, strategy, and organization. Amer. Econom. Rev. 80(3) 511-528.

Passariello, C. 2008. Logistics are in vogue with designers-as slump threatens luxury goods, systems to track consumer tastes and tweak offerings win converts. The Wall Street Journal.

Rohwedder, C., K. Johnson. 2008. Pace-setting Zara seeks more speed to fight its rising cheap-chic rivals. The Wall Street Journal.

Rozhon, T. 2004. Worried merchants throw discounts at shoppers. The New York Times.

Stokey, N. L. 1981. Rational expectations and durable goods pricing. The Bell Journal of Economics 12(1) $112-128$.

$\mathrm{Su}, \mathrm{X}$. 2007. Inter-temporal pricing and consumer stockpiling. Working paper, University of California, Berkeley.

$\mathrm{Su}$, X. 2009. Consumer returns policies and supply chain performance. Manufacturing Service Oper. Management 11(4) 595-612.

Su, X., F. Zhang. 2008. Strategic consumer behavior, commitment, and supply chain performance. Management Sci. 54(10) 1759-1773.

$\mathrm{Su}, \mathrm{X}$., F. Zhang. 2009. On the value of inventory information and availability guarantees when selling to strategic consumers. Management Sci. 55(5) 713-726.

Swinney, R. 2009. Selling to strategic consumers when product value is uncertain: The value of matching supply and demand. Working paper, Stanford University.

Yin, R., Y. Aviv, A. Pazgal, C. S. Tang. 2009. Optimal markdown pricing: Implications of inventory display formats in the presence of strategic customers. Management Sci. 55(8) 1391-1408. 